\title{
The Stellar Structures around Disk Galaxies
}

\author{
Igor Drozdovsky ${ }^{1,2}$, Nikolay Tikhonov ${ }^{3}$, Antonio Aparicio ${ }^{1,4}$, \\ Carme Gallart ${ }^{1}$, Matteo Monelli ${ }^{1}$, Sebastian Hidalgo ${ }^{1}$, \\ Edouard J. Bernard ${ }^{1}$, Olga Galazutdinova ${ }^{3}$ and the LCID team $\dagger$ \\ ${ }^{1}$ Instituto de Astrofísica de Canarias, Tenerife, Spain \\ (dio@iac.es; http://www.iac.es/galeria/dio) \\ ${ }^{2}$ Astronomical Institute of St.Petersburg State University, Russia \\ ${ }^{3}$ Special Astrophysical Observatory, Russia \\ ${ }^{4}$ Department of Astrophysics, University of La Laguna, Tenerife, Spain
}

\begin{abstract}
We present a brief summary of our current results on the stellar distribution and population gradients of the resolved stars in the surroundings of $\sim 50$ nearby disk galaxies, observed with space- (Hubble \& Spitzer) and ground-based telescopes (Subaru, VLT, BTA, Palomar, CFHT, \& INT). We examine the radial (in-plane) and vertical (extraplanar) distributions of resolved stars as a function of stellar age and metallicity by tracking changes in the color-magnitude diagram of face-on and edge-on galaxies. Our data show, that the scale length and height of a stellar population increases with age, with the oldest detected stellar populations identified at a large galactocentric radius or extraplanar height, out to typically a few kpc. In the most massive of the studied galaxies there is evidence for a break in number density and color gradients of evolved stars, which plausibly correspond to the thick disk and halo components of the galaxies. The ratio of intermediate-age to old stars in the outermost fields correlate with the gas fraction, while relative sizes of the thick-to-thin disks anticorrelate with galactic circular velocity.
\end{abstract}

Keywords. galaxies: dwarf, stellar content, structure; stars: imaging, statistics

Extended faint stellar structures have been detected around many nearby disk galaxies of different morphological types over the past decade, but their nature has been a matter of some debate. Sometimes referred either as a 'sheet'-like (thick disk), or a spherical 'halo' structure, these stellar formations together with streams are now recognized as an important probe of galaxy formation and evolution (e.g. Abadi et al. 2006). Using the multiband photometry of individual stars, we know that the scale length and height of a stellar population in the disk galaxies increase with age, with oldest detected stellar populations identified at large galactocentric radii or extraplanar height, out to typically a few kpc (e.g. Drozdovsky et al. 2003; Tikhonov, Galazutdinova \& Drozdovsky 2005; Tikhonov 2005, 2006). The extraplanar height of the thick disk in low mass disk galaxies is systematically larger than the young thin disk of giant spirals (see Fig. 1a,c), suggesting that stars in low-mass galaxies form in a thicker disk. In the most massive of the studied galaxies (highest circular velocity), there is evidence for a break in number density and color gradients of evolved Red Giant Branch (RGB) and Red Clump (RC) stars, which plausibly correspond to the transition from the thick disk to the elusive stellar spheroid, the halo.

As a next step in our study we selected four prominent Local Group disk galaxies, NGC 6822, NGC 3109, IC 1613, and IC 10 to analyze in-depth the radial (in-plane) and vertical (extraplanar) gradients as a function of stellar age in a wide range of

$\dagger$ Local Cosmology from Isolated Dwarfs; http://www.iac.es/project/LCID 

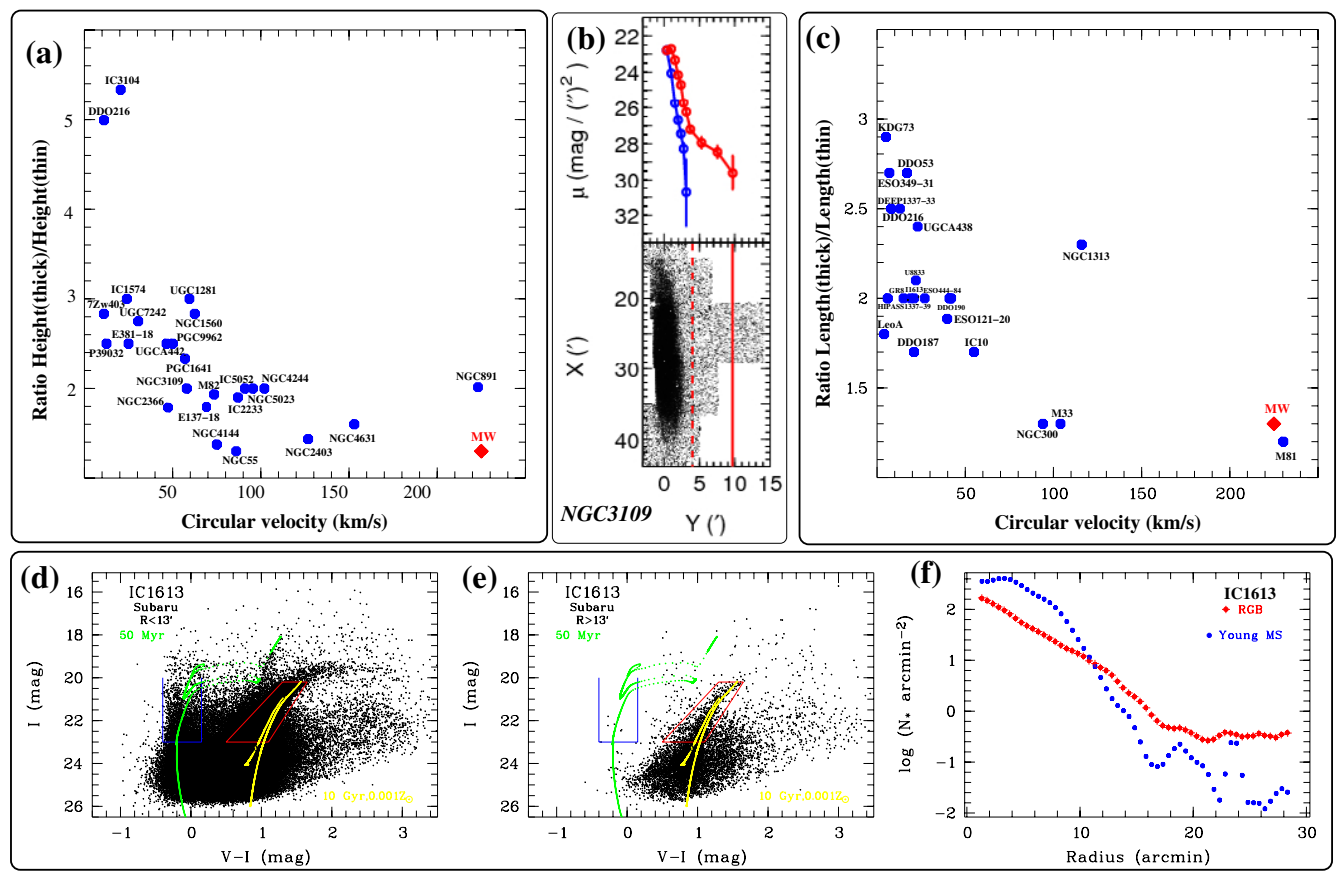

Figure 1. (a) Ratio of thick- to thin-disk height as a function of the rotational velocity for the high-inclination galaxies, based on the distribution of resolved young and evolved stars. An example of such study for the edge-on galaxy is shown on panel (b). (c)) The similar plot for the radial length ratio in the face-on sub-sample. Bottom panels: Results of our study of the stellar structures around IC1613, based on the data obtained with ground- (Subaru, CFHT, and INT) and space-based telescopes (HST \& Spitzer) in collaboration with the LCID project. (d), (e) are the CMDs for the inner and outer area, and $(f)$ is a radial population gradient.

galactocentric distances. Our data include wide-field imaging with ground-based telescopes aimed at reaching the old $(\sim 10$ Gyr $)$ stellar population, such as horizontal branch (HB) stars - signposts of an ancient stellar population-, and deep pencil beam fields with $\mathrm{HST} / \mathrm{ACS} \& W F P C 2$ reaching the old main-sequence turnoffs (based in part on the LCID team data). We are working on the modeling of the star formation history and chemical evolution of the different stellar sub-structures in these galaxies (see also Aparicio et al., Gallart et al., and Hidalgo et al., this meeting). Additionally, we are exploring the possibility to combine the optical data with near-infrared Spitzer/IRAC imaging to better disentangle the well-known age-metallicity degeneracy.

When complemented with detailed chemical abundances and kinematic information from the spectra of the individual stars, our optical/near-IR photometric data will allow us to shed light on fundamental questions about the evolution of disk galaxies, such as disk heating versus merger scenarios and the role of these mechanisms in forming the stellar disks and halos.

\section{References}

Abadi, M. G., Navarro, J. F., \& Steinmetz, M. 2006, MNRAS, 365, 747

Drozdovsky, I., Tikhonov, N., \& Schulte-Ladbeck, R. 2003, in "The Local Group as an Astrophysical Laboratory", 25

Tikhonov, N., Galazutdinova, O., \& Drozdovsky I., 2005, A\&AA, 431, 127.

Tikhonov, N., 2005, AstrRep, 49, 501; 2006, AstrRep, 50, 517 